\title{
Medical applications of shape memory alloys
}

\author{
Asha Kumawat*, Sanjay Kumawat and Mohammad Aarif \\ Mechanical Engineering Department, Poornima College of Engineering, \\ ISI-2, RIICO Institutional Area, Sitapura, Jaipur -302022 Rajasthan. \\ *Email: gavali.asha@gmail.com
}

\begin{abstract}
Shape memory alloys (SMA) constitute a group of metallic materials with the ability to recover a previously defined length or a shape when subjected to an appropriate thermo mechanical load. When there is a limitation of shape recovery, these alloys promote high Restitution forces. Because of these properties, there is a great technological interest in the use of SMA for different applications.
\end{abstract}

The first cardiovascular device developed with shape memory was the Simon filter. The Simon filter represents a new generation of devices that are used for blood vessel interruption in order to prevent pulmonary embolism. Persons who cannot take anticoagulant medicines are the major users of the Simon filter. The purpose of this device is to filter clots that travel inside the bloodstream. The Simon filter traps these clots that in time are dissolved by the bloodstream. The insertion of the filter inside the human body is done by exploiting the shape memory effect. From its original shape in the martensitic state the filter is deformed and placed on a catheter tip. Saline solution flowing through the catheter is used to keep a low temperature, while the filter is placed inside the body. When the catheter releases the filter, the flow of the saline solution is stopped.

As a result, the bloodstream promotes the heating of the filter that returns to its former shape. This procedure can be seen in Figure the atrial septal occlusion device is employed to seal the atrial hole. The atrial hole is located between the two upper heart chambers upon the surface that splits the upper part of the heart into the right and left atria. The anomaly occurring when this hole is open can reduce life expectancy.

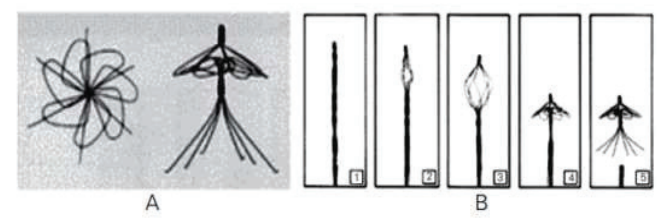

Figure 1: Simon filters. $A$, Filter in the recovery form. $B$, Filter release
The traditional surgery that fixes this anomaly is extremely invasive and dangerous. The thorax of the patient is opened and the atrial hole is sewn. Because of the intrinsic risks of this surgery, several problems might occur. The atrial septal occlusion device is an alternative to this surgery. This device is composed of SMA wires and a waterproof film of polyurethane. As is the case for the Simon filter, the surgery to place this device exploits the shape memory effect, being much less invasive than the traditional one.
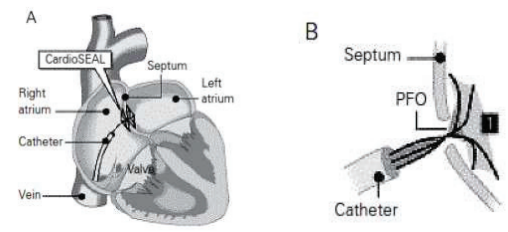

Figure 2: Atrial septal occlusion device.

Orthopedic applications: SMA has a large number of orthopedic applications. The spinal vertebra spacer is one. The insertion of this space between two vertebrae assures the local reinforcement of the spinal vertebrae, preventing any traumatic motion during the healing process. The use of a shape memory spacer permits the application of a constant load regardless of the position of the patient, who preserves some degree of motion.

Applications to surgical instruments: In recent years, medicine and the medical industry have focused on the concept of less invasive surgical procedures. Following this tendency, shape memory surgical instruments have been created and are becoming noticeable.

\section{References}

1. Hodgson DE, Wu MH \& Biermann RJ (1990). Shape Memory Alloys, Metals Handbook. Vol. 2. ASM International, Ohio, 897-902.

2. Mantovani D (2000). Shape memory alloys: Properties and biomedical applications. Journal of the Minerals, Metals and Materials Society, 52: 36-44.

3. Shape Memory Alloy Research Team (Smart) (2001). http:// smart.tamu.edu

4. Otsuka K \& Ren X (1999). Recent developments on the research of shape memory alloys. Intermetallics, 7: 511528. 